\title{
On Fuzzifying Nearly Compact Spaces
}

\author{
A.M. Zahran ${ }^{1}$, O.R. Sayed ${ }^{2}$, M. Azab Abd-Allah ${ }^{3}$ and A.K. Mousa ${ }^{4}$ \\ ${ }^{1,4}$ Department of Mathematics, Faculty of Science, Al-Azhar University, Assiut 71524, Egypt \\ ${ }^{2,3}$ Department of Mathematics, Faculty of Science, Assiut University, Assiut 71516, Egypt \\ E-mail : (o_r_sayed@yahoo.com)
}

\begin{abstract}
This paper considers fuzzifying topologies, a special case of $I$-fuzzy topologies (bifuzzy topologies) introduced by Ying $[16$, (I)]. It investigates topological notions defined by means of regular open sets when these are planted into the framework of Ying's fuzzifying topological spaces (in Łukasiewwicz fuzzy logic). The concept of fuzzifying nearly compact spaces is introduced and some of its properties are obtained. We use the finite intersection property to give a characterization of fuzzifying nearly compact spaces. Furthermore, we study the image of fuzzifying nearly compact spaces under fuzzifying completely continuous functions, fuzzifying almost continuity and fuzzifying R-map.
\end{abstract}

Key Words : Łukasiewicz logic; Fuzzifying topology; Fuzzifying regular open set; fuzzifying compact spaces; nearly compact spaces.

\section{Introduction}

Fuzzy topology, as an important research field in fuzzy set theory, has been developed into a quite mature discipline [4-5, 7, 9-10, 15]. In contrast to classical topology, fuzzy topology is endowed with richer structure, which is manifested with different ways to generalize certain classical concepts. So far, according to [5], the kind of topologies defined by Chang [1] and Goguen [2] is called the topologies of fuzzy subsets, and further is naturally called $L$-topological spaces if a lattice $L$ of membership values has been chosen. Loosely speaking, a topology of fuzzy subsets (resp. an $L$-topological space) is a family of fuzzy subsets (resp. $L$-fuzzy subsets) of nonempty set $X$, and satisfies the basic conditions of classical topologies [8]. On the other hand, the authors of $[6,11]$ proposed the terminologies $I$-fuzzy topologies (if the set of membership values is chosen to be the unit interval $[0,1])$ and $L$-fuzzy topologies (if the corresponding set of membership values is chosen to be lattice $L$ ). In [3], an $L$-fuzzy topology is an $L$-valued mapping on the traditional power set $P(X)$ of $X$. In [6, 9-11] an $L$-fuzzy topology is an $L$-valued mapping on the $L$-valued mapping on the $L$-power set of X. In 1980, Höhle [3] introduced the concept of fuzzy measurable spaces with the idea of giving degrees in $[0,1]$ to some topological terms rather than 0 and 1. In 1991, by Łukasiewicz logic on [0, 1], Ying [16] used the semantic method to propose so-called fuzzifying topology, whose definition is the same with Höhle's. He gave an elementally development of topology in the theory of fuzzy sets from a completely different direction. Briefly speaking, a fuzzifying topology on a set $X$ assigns each crisp subset of $X$ to a certain degree of being open, other than being definitely open or not. In fact, fuzzifying topologies are a special case of the $L$-fuzzy topologies in $[6,11]$ since all t-norm on $I$ are included as a special class of tensor products in these papers. Ying uses one particular tensor product, namely Łukasiewicz conjunction. Thus his fuzzifying topologies are a special class of all the $I$-fuzzy topologies considered in the categorical frameworks [6, 11]. Particularly, as the author [16] indicated, by investigating fuzzifying topology we may partially answer an important question proposed by Rosser and Turquette [12] in 1952, which asked whether there are many valued theories beyond the level of predicates calculus. Roughly speaking, the semantically analysis approach transforms formal statements of interest, which are usually expressed as implication formulas in logical language, into some inequalities in the truth value set by truth valuation rules, and then these inequalities are demonstrated in an algebraic way and the semantic validity of conclusions is established. In 1993, Ying [17] introduced the concepts of compactness and established a generalization of Tychonoff's theorem in the framework of fuzzifying topology. In [13-14] Singal and Mathur introduced the concept of nearly compact spaces in general topology. In this paper, we give the definitions and basic properties of fuzzifying open covering and fuzzifying nearly compact spaces. Also, we use the finite intersection property to give a characterization of fuzzifying nearly compact space.

Manuscript received Feb. 4, 2010; revised Jul. 8, 2010;

Accepted Oct. 21, 2010. 
Moreover, we study the image of fuzzifying nearly compact spaces under fuzzifying completely continuous functions, fuzzifying almost continuity and fuzzifying R-map. We use the terminologies and notations in [16-19] without any explanation. We will use the symbol $\otimes$ instead of the second "AND" operation $\wedge$ as the dot is hardly visible. This means that $[\alpha] \leq[\varphi \rightarrow \dot{\psi}] \Leftrightarrow[\alpha] \otimes[\varphi] \leq[\psi]$. Also, we need the fact that $(\alpha \rightarrow \gamma) \wedge(\beta \rightarrow \gamma)=(\alpha \vee \beta) \rightarrow \gamma$. A fuzzifying topology on a set $X[3,16]$ is a function $\tau \in \Im(P(X))$ such that:

(1) $\tau(X)=\tau(\emptyset)=1$;

(2) for any $A, B \in P(X), \tau(A \cap B) \geq \tau(A) \wedge \tau(B)$;

(3) for any $\left\{A_{\lambda} \in P(X): \lambda \in \Lambda\right\}, \tau\left(\bigcup_{\lambda \in \Lambda} A_{\lambda}\right) \geq$ $\bigwedge_{\lambda \in \Lambda} \tau\left(A_{\lambda}\right)$.

The family of all fuzzifying regular open sets [18], denoted by

$\tau_{R} \in \Im(P(X))$, is defined as follows:

$$
\begin{aligned}
& {\left[A \in \tau_{R}\right]=\min }\left(\bigwedge_{x \in A}(\operatorname{Int}(C l(A)(x)),\right. \\
&\left.\bigwedge_{x \in X-A}(1-\operatorname{Int}(C l(A))(x))\right) .
\end{aligned}
$$

If $(X, \tau)$ and $(Y, \sigma)$ are two fuzzifying topological spaces and $f \in Y^{X}$. Then, the unary fuzzy predicates $A C, C_{C}$ and $C_{R} \in \Im\left(Y^{X}\right)$ called fuzzifying almost continuity [18], fuzzifying completely continuous function [19] and fuzzifying R-map [19], are given respectively, as follows: $A C(f):=\forall U\left(U \in \sigma_{R} \longrightarrow f^{-1}(U) \in \tau\right)$,

$C_{C}(f):=(\forall U)\left(U \in \sigma \longrightarrow f^{-1}(U) \in \tau_{R}\right)$, and $C_{R}(f):=(\forall U)\left(U \in \sigma_{R} \longrightarrow f^{-1}(U) \in \tau_{R}\right)$.

If $\Omega$ is the class of all fuzzifying topological spaces, then a unary fuzzy predicate $\Gamma \in \Im(\Omega)$, called fuzzifying compactness [17], is given as follows:

(1) $\Gamma(X, \tau):=(\forall \Re)\left(K_{\circ}(\Re, X) \longrightarrow(\exists \wp)((\wp \leq\right.$ $\Re) \wedge K(\wp, X) \otimes F F(\wp)))$.

(for $K$ and $K_{\circ}$ see [16,(II)], Definition 4.4, for $\leq$ see [16,(II)], Theorem 4.3 and for $F F$ see [17], Definition 1.1)

(2) If $A \subseteq X$, then $\Gamma(A):=\Gamma(A, \tau / A)$

\section{Fuzzifying nearly compact space}

\section{Definition 2.1}

(1) A binary fuzzy predicate $K_{R} \in \Im(\Im(P(X)) \times$ $P(X)$ ), called fuzzifying regular open covering, is given as follows:

$$
K_{R}(\Re, A):=K(\Re, A) \otimes\left(\Re \subseteq \tau_{R}\right) .
$$

(2) Let $\Omega$ be the class of all fuzzifying topological spaces. A unary fuzzy predicates $\Gamma_{R} \in \Im(\Omega)$, called fuzzifying near compactness, is given as follows:

(i) $\Gamma_{R}(X, \tau):=(\forall \Re)\left(K_{R}(\Re, X) \longrightarrow(\exists \wp)((\wp \leq\right.$
$\Re) \wedge K(\wp, X) \otimes F F(\wp)))$

(ii) If $A \subseteq X$, then $\Gamma_{R}(A):=\Gamma_{R}(A, \tau / A)$;

(3) Let $(X, \tau)$ be a fuzzifying topological space and $A \subseteq X$, then the family of all fuzzifying regular open sets in $(A, \tau / A)$ denoted by $\tau_{R} / A \in \Im(P(A))$ is defined as

$$
V \in \tau_{R} / A:=(\exists U)\left(\left(U \in \tau_{R}\right) \wedge(V=U \cap A)\right) .
$$

The following theorem states that "the degree to which a subset $A$ is fuzzifying nearly compact is equal to the degree in the statement "every fuzzifying regular open cover from $\tau$ of $A$ has a finite subcover".

Theorem 2.2. For any fuzzifying topological space $(X, \tau)$ and $A \subseteq X$,

$\vDash \Gamma_{R}(A) \longleftrightarrow(\forall \Re)\left(K_{R}(\Re, A) \longrightarrow(\exists \wp)((\wp \leq\right.$ $\Re) \wedge K(\wp, A) \otimes F F(\wp)))$,

where $K_{R}$ is related to $\tau$.

Proof. For any $\Re \in \Im(P(X))$, we set $\bar{\Re} \in \Im(P(A))$ defined as

$$
\bar{\Re}(C)=\bigvee_{C=A \cap B, B \subseteq X} \Re(B) .
$$

Then

$$
\begin{aligned}
{[K(\bar{\Re}, A)] } & =\bigwedge_{x \in A} \bigvee_{x \in C} \bar{\Re}(C) \\
& \geq \bigwedge_{x \in A} \bigvee_{x \in C=A \cap B} \Re(B) \\
& \geq \bigwedge_{x \in A} \bigvee_{x \in B} \Re(B)=[K(\Re, A)],
\end{aligned}
$$

Therefore

$$
\begin{aligned}
& {\left[\bar{\Re} \subseteq \tau_{R} / A\right]} \\
& =\bigwedge_{C \subseteq A} \min \left(1,1-\bar{\Re}(C)+\tau_{R} / A(C)\right) \\
& =\bigwedge_{C \subseteq A} \min \left(1,1-\bigvee_{C=A \cap B, B \subseteq X} \Re(B)+\bigvee_{C=A \cap B, B \subseteq X} \tau_{R}(B)\right) \\
& \geq \bigwedge_{C \subseteq A, C=A \cap B, B \subseteq X} \min \left(1,1-\Re(B)+\tau_{R}(B)\right) \\
& \geq \bigwedge_{B \subseteq X} \min \left(1,1-\Re(B)+\tau_{R}(B)\right)=\left[\Re \subseteq \tau_{R}\right] .
\end{aligned}
$$

Now, we define $\left[K_{R}(\Re, A)\right]=\left[K(\Re, A) \otimes\left(\Re \subseteq \tau_{R}\right)\right]$ and $\left[K_{R}^{\prime}(\bar{\Re}, A)\right]=\left[K(\bar{\Re}, A) \otimes\left(\bar{\Re} \subseteq \tau_{R} / A\right)\right]$. Then $\left[K_{R}(\Re, A)\right] \leq\left[K_{R}^{\prime}(\bar{\Re}, A)\right]$.

So, for any $\wp \leq \bar{\Re}$, we define $\wp^{\prime} \in \Im(P(X))$ as

$$
\wp^{\prime}(B)= \begin{cases}\wp(B), & \text { if } B \subseteq A \\ 0, & \text { otherwise. }\end{cases}
$$

Then $\wp^{\prime} \leq \Re, \quad\left[F F\left(\wp^{\prime}\right)\right]=[F F(\wp)]$ and $\left[K\left(\wp^{\prime}, A\right)\right]=$ 
$[K(\wp, A)]$. Furthermore, we have

$$
\begin{aligned}
& {\left[\Gamma_{R}(A) \otimes K_{R}(\Re, A)\right]} \\
& \leq\left[\Gamma_{R}(A) \otimes K_{R}^{\prime}(\bar{\Re}, A)\right] \\
& =\left[( \forall \overline { \Re } ) \left(K_{R}^{\prime}(\bar{\Re}, A) \longrightarrow(\exists \wp)((\wp \leq \bar{\Re}) \wedge K(\wp, A)\right.\right. \\
& \left.\quad \otimes F F(\wp))) \otimes K_{R}^{\prime}(\bar{\Re}, A)\right] \\
& \leq\left[K_{R}^{\prime}(\bar{\Re}, A) \longrightarrow(\exists \wp)((\wp \leq \bar{\Re}) \wedge K(\wp, A)\right. \\
& \left.\quad \quad \otimes F F(\wp)) \otimes K_{R}^{\prime}(\bar{\Re}, A)\right] \\
& \leq[(\exists \wp)((\wp \leq \bar{\Re}) \wedge K(\wp, A) \otimes F F(\wp))] \\
& \leq\left[\left(\exists \wp^{\prime}\right)\left(\left(\wp^{\prime} \leq \Re\right) \wedge K\left(\wp^{\prime}, A\right) \otimes F F\left(\wp^{\prime}\right)\right)\right] \\
& \leq[(\exists ß)((ß \leq \Re) \wedge K(ß, A) \otimes F F(ß))] .
\end{aligned}
$$

Then $\left[\Gamma_{R}(A)\right] \leq\left[K_{R}(\Re, A)\right] \longrightarrow[(\exists ß)((\beta \leq \Re) \wedge$ $K(B, A) \otimes F F(B))]$. Therefore

$$
\begin{gathered}
{\left[\Gamma_{R}(A)\right] \leq \bigwedge_{\Re \in \Im(P(X))}\left[K_{R}(\Re, A) \longrightarrow(\exists ß)((\beta \leq \Re)\right.} \\
\wedge K(\beta, A) \otimes F F(\beta))] \\
=\left[( \forall \Re ) \left(K_{R}(\Re, A) \longrightarrow(\exists \beta)((\beta \leq \Re)\right.\right. \\
\wedge K(ß, A) \otimes F F(\beta)))] .
\end{gathered}
$$

Conversely, for any $\Re \in \Im(P(A))$, if $\left[\Re \subseteq \tau_{R} / A\right]=$ $\bigwedge_{B \subseteq A} \min \left(1,1-\Re(B)+\tau_{R} / A(B)\right)=\lambda$, then for any $n \in N$ and $B \subseteq A, \quad \bigvee_{B=A \cap C, C \subseteq X} \tau_{R}(C)=\tau_{R} / A(B)>$ $\lambda+\Re(B)-1-\frac{1}{n}$, and there exists $C_{B} \subseteq X$ such that $C_{B} \cap A=B$ and $\tau_{R}\left(C_{B}\right)>\lambda+\Re(B)-1-\frac{1}{n}$. Now, we define $\bar{\Re} \in \Im(P(X))$ as $\bar{\Re}(C)=\max _{B \subseteq A}\left(0, \lambda+\Re(B)-1-\frac{1}{n}\right)$. Then $\left[\bar{\Re} \subseteq \tau_{R}\right]=1$ and

$$
\begin{aligned}
{[K(\bar{\Re}, A)] } & =\bigwedge_{x \in A} \bigvee_{x \in C \subseteq X} \bar{\Re}(C) \\
& =\bigwedge_{x \in A} \bigvee_{x \in B} \bar{\Re}\left(C_{B}\right) \\
& \geq \bigwedge_{x \in A} \bigvee_{x \in B}\left(\lambda+\Re(B)-1-\frac{1}{n}\right) \\
& =\bigwedge_{x \in A} \bigvee_{x \in B} \Re(B)+\lambda-1-\frac{1}{n} \\
& =K(\Re, A)+\lambda-1-\frac{1}{n},
\end{aligned}
$$

$\left[K_{R}(\bar{\Re}, A)\right]$

$=\left[K(\bar{\Re}, A) \otimes\left(\bar{\Re} \subseteq \tau_{R}\right)\right]$

$=[K(\bar{\Re}, A)] \geq \max \left(0, K(\Re, A)+\lambda-1-\frac{1}{n}\right)$

$\geq \max (0, K(\Re, A)+\lambda-1)-\frac{1}{n}=K_{R}^{\prime}(\Re, A)-\frac{1}{n}$.

For any $\wp \leq \bar{\Re}$, we set $\wp^{\prime} \in \Im(P(A))$ as $\wp^{\prime}(B)=$ $\wp\left(C_{B}\right), B \subseteq A$.
Then $\wp^{\prime} \leq \Re, \quad\left[F F\left(\wp^{\prime}\right)\right]=[F F(\wp)]$ and $\left[K\left(\wp^{\prime}, A\right)\right]=$ $[K(\wp, A)]$. Therefore

$$
\begin{aligned}
& {\left[( \forall \Re ) \left(K_{R}(\Re, A) \longrightarrow(\exists \wp)((\wp \leq \Re) \wedge K(\wp, A)\right.\right.} \\
& \otimes F F(\wp)))] \otimes\left[K_{R}^{\prime}(\Re, A)\right]-\frac{1}{n} \\
& \leq\left[( \forall \Re ) \left(K_{R}(\Re, A) \longrightarrow(\exists \wp)((\wp \leq \Re) \wedge K(\wp, A)\right.\right. \\
& \otimes F F(\wp)))] \otimes\left(\left[K_{R}^{\prime}(\Re, A)\right]-\frac{1}{n}\right) \\
& \leq\left[K_{R}(\bar{\Re}, A) \longrightarrow(\exists \wp)((\wp \leq \bar{\Re}) \wedge K(\wp, A)\right. \\
& \otimes F F(\wp))] \otimes\left[K_{R}(\bar{\Re}, A)\right] \\
& \leq[(\exists \wp)((\wp \leq \bar{\Re}) \wedge K(\wp, A) \otimes F F(\wp))] \\
& \leq\left[\left(\exists \wp^{\prime}\right)\left(\left(\wp^{\prime} \leq \Re\right) \wedge K\left(\wp^{\prime}, A\right) \otimes F F\left(\wp^{\prime}\right)\right)\right] \\
& \leq[(\exists ß)((\beta \leq \Re) \wedge K(\beta, A) \otimes F F(\beta))] .
\end{aligned}
$$

Let $n \longrightarrow \infty$. We obtain

$$
\begin{aligned}
& {\left[( \forall \Re ) \left(K_{R}(\Re, A) \longrightarrow(\exists \wp)((\wp \leq \Re) \wedge K(\wp, A)\right.\right.} \\
& \otimes F F(\wp))] \otimes\left[K_{R}^{\prime}(\Re, A)\right] \\
& \leq[(\exists ß)((\Re \leq \Re) \wedge K(\Re, A) \otimes F F(\beta))] . \text { Then } \\
& {\left[(\forall \Re)\left(K_{R}(\Re, A) \longrightarrow(\exists \wp)((\wp \leq \Re) \wedge K(\wp, A) \otimes F F(\wp))\right)\right]} \\
& \leq\left[K_{R}^{\prime}(\Re, A) \longrightarrow(\exists ß)((ß \leq \Re) \wedge K(\Re, A) \otimes F F(\beta))\right] \\
& \leq \bigwedge_{\Re \in \Im(P(X))}\left[K_{R}^{\prime}(\Re, A) \longrightarrow(\exists \beta)((\beta \leq \Re) \wedge K(\beta, A)\right. \\
& \otimes F F(ß))]=\left[\Gamma_{R}(A)\right] .
\end{aligned}
$$

In the following theorem we give a characterization of fuzzifying nearly compact space by using the finite intersection property.

\section{Theorem 2.3.}

Let $(X, \tau)$ be a fuzzifying topological space and let $\pi_{1}:=(\forall \Re)\left((\Re \in \Im(P(X))) \wedge\left(\Re \subseteq F_{R}\right) \otimes \mathrm{fI}(\Re) \longrightarrow\right.$ $(\exists x)(\forall A)(A \in \Re \longrightarrow x \in A))$;

$\pi_{2}:=(\forall \Re)(\exists B)\left(\left(\left(\Re \subseteq F_{R}\right) \wedge\left(B \in \tau_{R}\right)\right) \otimes\right.$ $(\forall \wp)((\wp \leq \Re) \otimes F F(\wp) \longrightarrow \neg(\bigcap \wp \subseteq B)) \longrightarrow$ $\neg(\bigcap \Re \subseteq B))$.

Then $=\Gamma_{R}(X, \tau) \longleftrightarrow \pi_{i}, i=1,2$. (for fI see [17], Definition 2.2)

Proof. (a) First, we prove $\left[\Gamma_{R}(X, \tau)\right]=\left[\pi_{1}\right]$.

For any $\Re \in \Im(P(X))$, we set $\Re^{c}(X-A)=\Re(A)$. Then

$\left[\Re \subseteq \tau_{R}\right]$

$=\bigwedge_{A \in P(X)} \min \left(1,1-\Re(A)+\tau_{R}(A)\right)$

$=\bigwedge_{X-A \in P(X)} \min \left(1,1-\Re^{c}(X-A)+F_{R}(X-A)\right)$

$=\left[\Re^{c} \subseteq F_{R}\right]$,

$[F F(\Re)]=1-\bigwedge\left\{\alpha \in[0,1]: F\left(\Re_{\alpha}\right)\right\}=1-\bigwedge\{\alpha \in$ $\left.[0,1]: F\left(\Re_{\alpha}^{c}\right)\right\}=\left[F F\left(\Re^{c}\right)\right]$ and

$\beta \leq \Re^{c} \longleftrightarrow B(A) \leq \Re^{c}(A) \longleftrightarrow \beta^{c}(X-A)$

$\leq \Re(X-A) \longleftrightarrow \beta^{c} \leq \Re$. 
Therefore

$$
\begin{aligned}
& {\left[\Gamma_{R}(X, \tau)\right]} \\
& =\left[( \forall \Re ) \left(K_{R}(\Re, X) \longrightarrow(\exists \wp)((\wp \leq \Re)\right.\right. \\
& \wedge K(\wp, X) \otimes F F(\wp)))] \\
& =\left[( \forall \Re ) \left(\left(\Re \subseteq \tau_{R}\right) \otimes K(\Re, X) \longrightarrow\right.\right. \\
& (\exists \wp)((\wp \leq \Re) \wedge K(\wp, X) \otimes F F(\wp)))] \\
& =\left[( \forall \Re ) \left(\left(\Re \subseteq \tau_{R}\right) \longrightarrow(K(\Re, X) \longrightarrow\right.\right. \\
& (\exists \wp)((\wp \leq \Re) \wedge K(\wp, X) \otimes F F(\wp))))] \\
& =\left[( \forall \Re ) \left(\left(\Re^{c} \subseteq F_{R}\right) \longrightarrow((\forall x)(\exists A)(A \in \Re \wedge x \in A)\right.\right. \\
& \longrightarrow(\exists \wp)((\wp \leq \Re) \wedge K(\wp, X) \otimes F F(\wp)))] \\
& =\left[( \forall \Re ) \left(\left(\Re^{c} \subseteq F_{R}\right) \longrightarrow((\forall x)(\exists A)(A \in \Re \wedge x \in A)\right.\right. \\
& \left.\left.\longrightarrow\left(\exists \beta^{c}\right)\left(\left(\Re^{c} \leq \Re\right) \wedge K\left(\Re^{c}, X\right) \otimes F F\left(\Re^{c}\right)\right)\right)\right] \\
& =\left[( \forall \Re ) \left(\left(\Re^{c} \subseteq F_{R}\right) \longrightarrow((\forall x)(\exists A)(A \in \Re \wedge x \in A)\right.\right. \\
& \left.\left.\longrightarrow(\exists ß)\left(\left(ß \leq \Re^{c}\right) \wedge F F(\beta) \otimes K\left(\Re^{c}, X\right)\right)\right)\right] \\
& =\left[( \forall \Re ) \left(\left(\Re^{c} \subseteq F_{R}\right) \longrightarrow((\forall x)(\exists A)(A \in \Re \wedge x \in A)\right.\right. \\
& \longrightarrow(\exists ß)\left(\left(\beta \leq \Re^{c}\right) \wedge F F(ß)\right. \\
& \left.\left.\left.\otimes(\forall x)(\exists B)\left(B \in \beta^{c} \wedge x \in B\right)\right)\right)\right] \\
& =\left[( \forall \Re ) \left(( \Re ^ { c } \subseteq F _ { R } ) \longrightarrow \left(\neg \left(( \exists ß ) \left(\left(ß \leq \Re^{c}\right) \wedge F F(ß)\right.\right.\right.\right.\right. \\
& \left.\left.\otimes(\forall x)(\exists B)\left(B \in B^{c} \wedge x \in B\right)\right)\right) \\
& \longrightarrow \neg((\forall x)(\exists A)(A \in \Re \wedge x \in A))))] \\
& =\left[( \forall \Re ) \left(( \Re ^ { c } \subseteq F _ { R } ) \longrightarrow \left(\mathrm{fI}\left(\Re^{c}\right) \longrightarrow\right.\right.\right. \\
& \neg((\forall x)(\exists A)(A \in \Re \wedge x \in A))))] \\
& =\left[( \forall \Re ) \left(\left(\Re^{c} \subseteq F_{R}\right) \otimes \mathrm{fI}\left(\Re^{c}\right) \longrightarrow\right.\right. \\
& \left.\left.(\exists x)(\forall A)\left(A \in \Re^{c} \wedge x \in A\right)\right)\right]=\left[\pi_{1}\right] .
\end{aligned}
$$

(b) We prove $\left[\pi_{1}\right]=\left[\pi_{2}\right]$. Let $X-B \in P(X)$. For any $\Re \in \Im(P(X))$, we have

$$
\begin{aligned}
& {\left[\left(\Re \subseteq F_{R}\right) \wedge\left(B \in \tau_{R}\right)\right] } \\
= & {\left[\left(\Re \subseteq F_{R}\right) \wedge\left(X-B \in F_{R}\right)\right] } \\
= & \bigwedge_{A \in P(X)} \min \left(1,1-\Re(A)+F_{R}(A)\right) \wedge F_{R}(X-B) \\
= & \bigwedge_{A \in P(X)} \min \left(1,1-\Re(A)+F_{R}(A)\right) \wedge \\
& \bigwedge_{A \in P(X)} \min \left(1,1-[A \in\{X-B\}]+F_{R}(A)\right) \\
= & \bigwedge_{A \in P(X)} \min \left(1,1-[(\Re \cup\{X-B\})(A)]+F_{R}(A)\right) \\
= & {\left[(\Re \cup\{X-B\}) \subseteq F_{R}\right] . }
\end{aligned}
$$

Therefore, for any $\beta \in \Im(P(X))$, let $\wp=ß \mid\{X-B\} \in \Im(P(X))$.

$$
\wp(A)= \begin{cases}\beta(A), & \text { if } A \neq X-B \\ 0, & \text { if } A=X-B\end{cases}
$$

Then $\wp \leq \beta, \wp \cup\{X-B\} \geq \beta, \quad[F F(\wp)]=$
$[F F(ß)],[\wp \leq \Re]=[ß \leq(\Re \cup\{X-B\})]$ and

$$
\begin{aligned}
& {[(\forall \wp)((\wp \leq \Re) \otimes F F(\wp) \longrightarrow} \\
& (\exists x)(\forall A)(A \in(\wp \cup\{X-B\}) \longrightarrow x \in A))] \\
= & \bigwedge_{\wp \leq \Re} \min (1,1-[F F(\wp)]+ \\
& \left.\bigvee_{x \in X} \bigwedge_{A \in P(X)}((\wp \cup\{X-B\})(A) \longrightarrow A(x))\right) \\
\leq & \bigwedge_{B \leq(\Re \cup\{X-B\})} \min (1,1-[F F(B)]+ \\
& \left.\bigvee_{x \in X} \bigwedge_{A \in P(X)}(B(A) \longrightarrow A(x))\right) \\
= & \mathrm{fI}(\Re \cup\{X-B\}) .
\end{aligned}
$$

Furthermore, we have

$$
\begin{aligned}
& {\left[\pi_{1}\right] \otimes\left[\left(\left(\Re \subseteq F_{R}\right) \wedge\left(B \in \tau_{R}\right)\right) \otimes(\forall \wp)((\wp \leq \Re)\right.} \\
& \otimes F F(\wp) \longrightarrow \neg(\bigcap \wp \subseteq B))] \\
& =\left[\pi_{1}\right] \otimes\left[\left(\Re \cup\{X-B\} \subseteq F_{R}\right) \otimes(\forall \wp)((\wp \leq \Re) \otimes\right. \\
& F F(\wp) \longrightarrow(\exists x)(\forall A)(A \in(\wp \cup\{X-B\}) \longrightarrow x \in A))] \\
& =\left[\pi_{1}\right] \otimes\left[\left(\Re \cup\{X-B\} \subseteq F_{R}\right) \otimes \mathrm{fI}(\Re \cup\{X-B\})\right] \\
& \leq[(\exists x)(\forall A)(A \in(\Re \cup\{X-B\}) \longrightarrow x \in A)] \\
& =[\neg(\bigcap \Re \subseteq B)] .
\end{aligned}
$$

\section{Therefore}

$$
\begin{aligned}
{\left[\pi_{1}\right] \leq } & \bigwedge_{\Re \in \Im(P(X))} \bigvee_{B \subseteq X}\left(\left(\left(\Re \subseteq F_{R}\right) \wedge\left(B \in \tau_{R}\right)\right) \otimes\right. \\
& (\forall \wp)((\wp \leq \Re) \otimes F F(\wp) \longrightarrow \\
& \neg(\bigcap \wp \subseteq B)) \longrightarrow \neg(\bigcap \Re \subseteq B))=\left[\pi_{2}\right] .
\end{aligned}
$$

Conversely,

$$
\begin{aligned}
& {\left[\pi_{2}\right] \otimes\left[\left(\Re \subseteq F_{R}\right) \otimes \mathrm{fI}(\Re)\right]} \\
& =\left[\pi_{2}\right] \otimes\left[((\Re \mid\{B\}) \cup\{B\}) \subseteq F_{R}\right) \\
& \otimes \mathrm{fI}((\Re \mid\{B\}) \cup\{B\})] \\
& =\left[\pi_{2}\right] \otimes\left[\left(\Re \Re^{\prime} \subseteq F_{R}\right) \wedge\left(X-B \in \tau_{R}\right)\right. \\
& \otimes(\forall \wp)\left(\wp \leq \Re^{\prime} \otimes F F(\wp) \longrightarrow\right. \\
& \quad(\exists x)(\forall A)(A \in(\wp \cup\{B\}) \longrightarrow x \in A))] \\
& =\left[\pi_{2}\right] \otimes\left[\left(\Re \Re^{\prime} \subseteq F_{R}\right) \wedge\left(X-B \in \tau_{R}\right)\right. \\
& \quad \otimes(\forall \wp)\left(\wp \leq \Re^{\prime} \otimes F F(\wp) \longrightarrow\right. \\
& \quad \neg(\bigcap \wp \subseteq X-B))] \\
& \leq[\neg(\bigcap \Re \subseteq X-B)] \\
& =\left[(\exists x)(\forall A)\left(A \in\left(\Re^{\prime} \cup\{B\}\right) \longrightarrow x \in A\right)\right] \\
& =[(\exists x)(\forall A)(A \in \Re \longrightarrow x \in A)] .
\end{aligned}
$$


Therefore

$$
\begin{aligned}
{\left[\pi_{2}\right] \leq } & \bigwedge_{\Re \in \Im(P(X))}\left[\left(\Re \subseteq F_{R}\right) \otimes \mathrm{fI}(\Re) \longrightarrow\right. \\
& (\exists x)(\forall A)(A \in \Re \longrightarrow x \in A)]=\left[\pi_{1}\right] .
\end{aligned}
$$

\section{Some properties of fuzzifying nearly compact spaces}

The following theorem gives the image of fuzzifying nearly compact space under fuzzifying completely continuous function.

Theorem 3.1. Let $(X, \tau)$ and $(Y, \sigma)$ be any two fuzzifying topological spaces and $f \in Y^{X}$ be surjection. Then $=\Gamma_{R}(X, \tau) \otimes C_{C}(f) \longrightarrow \Gamma(f(X))$.

Proof. For any $\bar{\Re} \in \Im(P(Y))$, we define $\Re \in \Im(P(X))$ as $\Re(A)=f^{-1}(\bar{\Re})(A)=\bar{\Re}(f(A))$. Then

$$
\begin{aligned}
{[K(\Re, X)] } & =\bigwedge_{x \in X} \bigvee_{x \in A} \Re(A)=\bigwedge_{x \in X} \bigvee_{x \in A} \bar{\Re}(f(A)) \\
& =\bigwedge_{x \in X} \bigvee_{f(x) \in B} \bar{\Re}(B) \\
& =\bigwedge_{y \in f(X)} \bigvee_{y \in B} \bar{\Re}(B)=[K(\bar{\Re}, f(X))],
\end{aligned}
$$

$[\bar{\Re} \subseteq \sigma] \otimes\left[C_{C}(f)\right]$

$=\bigwedge_{B \subseteq Y} \min (1,1-\bar{\Re}(B)+\sigma(B))$

$$
\begin{gathered}
\otimes \bigwedge_{B \subseteq Y} \min \left(1,1-\sigma(B)+\tau_{R}\left(f^{-1}(B)\right)\right) \\
=\max \left(0, \bigwedge_{B \subseteq Y} \min (1,1-\bar{\Re}(B)+\sigma(B))\right. \\
\left.+\bigwedge_{B \subseteq Y} \min \left(1,1-\sigma(B)+\tau_{R}\left(f^{-1}(B)\right)\right)-1\right)
\end{gathered}
$$$$
\leq \bigwedge_{B \subseteq Y} \max (0, \min (1,1-\bar{\Re}(B)+\sigma(B))
$$$$
\left.+\min \left(1,1-\sigma(B)+\tau_{R}\left(f^{-1}(B)\right)\right)-1\right)
$$$$
\leq \bigwedge_{B \subseteq Y} \min \left(1,1-\bar{\Re}(B)+\tau_{R}\left(f^{-1}(B)\right)\right)
$$$$
=\bigwedge_{A \subseteq X} \bigwedge_{f^{-1}(B)=A} \min \left(1,1-\bar{\Re}(B)+\tau_{R}\left(f^{-1}(B)\right)\right)
$$$$
=\bigwedge_{A \subseteq X} \bigwedge_{f^{-1}(B)=A} \min \left(1,1-\bar{\Re}(B)+\tau_{R}(A)\right)
$$$$
=\bigwedge_{A \subseteq X} \min \left(1,1-\bigvee_{f^{-1}(B)=A} \bar{\Re}(B)+\tau_{R}(A)\right)
$$$$
=\bigwedge_{A \subseteq X} \min \left(1,1-\Re(A)+\tau_{R}(A)\right)=\left[\Re \subseteq \tau_{R}\right] .
$$

For any $\wp \leq \Re$, we set $\bar{\varsigma} \in \Im(P(Y))$ defined as $\bar{\wp}(f(A))=f(\wp)(f(A))=\wp(A), A \subseteq X$.

$$
\text { Then } \quad \begin{aligned}
\bar{\wp}(f(A)) & =f(\wp)(f(A)) \\
& \leq f(\Re)(f(A)) \\
& =f\left(f^{-1}(\bar{\Re})(f(A))\right) \leq \bar{\Re}(f(A)),
\end{aligned}
$$

$[F F(\wp)]=1-\bigwedge\{\alpha \in[0,1]: F(\wp[\alpha])\} \leq 1-\bigwedge\{\alpha \in$ $\left.[0,1]: F\left(f(\wp)_{[\alpha]}\right)\right\}=[F F(f(\wp))]=[F F(\bar{\wp})]$ and

$$
\begin{aligned}
{[K(\bar{\wp}, f(X))] } & =\bigwedge_{y \in f(X)} \bigvee_{y \in B} \bar{\wp}(B) \\
& =\bigwedge_{y \in f(X)} \bigvee_{y \in B=f(A)} \wp(A) \\
& \geq \bigwedge_{y \in f(X)} \bigvee_{f^{-1}(y) \in A} \wp(A) \\
& =\bigwedge_{x \in X} \bigvee_{x \in A} \wp(A)=[K(\wp, X)]
\end{aligned}
$$

Furthermore

$$
\begin{aligned}
& {\left[\Gamma_{R}(X, \tau)\right] \otimes\left[C_{C}(f)\right] \otimes\left[K_{\circ}^{\prime}(\bar{\Re}, f(X))\right]} \\
& =\left[\Gamma_{R}(X, \tau)\right] \otimes\left[C_{C}(f)\right] \otimes[K(\bar{\Re}, f(X))] \otimes[\bar{\Re} \subseteq \sigma] \\
& \leq\left[\Gamma_{R}(X, \tau)\right] \otimes\left[\Re \subseteq \tau_{R}\right] \otimes[K(\Re, X)] \\
& =\left[\Gamma_{R}(X, \tau)\right] \otimes\left[K_{R}(\Re, X)\right] \\
& \leq[(\exists \wp)((\wp \leq \Re) \wedge K(\wp, X) \otimes F F(\wp))] \\
& \leq[(\exists \wp)((\wp \leq \Re) \wedge K(\bar{\wp}, f(X)) \otimes F F(\bar{\wp}))] \\
& \leq\left[\left(\exists \wp^{\prime}\right)\left(\left(\wp^{\prime} \leq \bar{\Re}\right) \wedge K\left(\wp^{\prime}, f(X)\right) \otimes F F\left(\wp^{\prime}\right)\right)\right],
\end{aligned}
$$

where $K_{\circ}^{\prime}$ is related to $\sigma$. Therefore

$$
\begin{aligned}
& {\left[\Gamma_{R}(X, \tau)\right] \otimes\left[C_{C}(f)\right] } \\
\leq & K_{\circ}^{\prime}(\bar{\Re}, f(X)) \longrightarrow\left(\exists \wp^{\prime}\right)\left(\left(\wp^{\prime} \leq \bar{\Re}\right)\right. \\
& \left.\wedge K\left(\wp^{\prime}, f(X)\right) \otimes F F\left(\wp^{\prime}\right)\right) \\
\leq & \bigwedge_{\bar{\Re} \in \Im(P(Y))}\left(K _ { \circ } ^ { \prime } ( \overline { \Re } , f ( X ) ) \longrightarrow ( \exists \wp ^ { \prime } ) \left(\left(\wp^{\prime} \leq \bar{\Re}\right)\right.\right. \\
& \left.\left.\wedge K\left(\wp^{\prime}, f(X)\right) \otimes F F\left(\wp^{\prime}\right)\right)\right) \\
= & {[\Gamma(f(X))] . \square }
\end{aligned}
$$

The following theorem gives the preservation of fuzzifying nearly compact space under fuzzifying R-map.

Theorem 3.2. Let $(X, \tau)$ and $(Y, \sigma)$ be any two fuzzifying topological spaces and $f \in Y^{X}$ be surjection. Then $\models \Gamma_{R}(X, \tau) \otimes C_{R}(f) \longrightarrow \Gamma_{R}(f(X))$.

Proof. From the proof of Theorem 3.1 we have for any $\bar{\Re} \in \Im(P(Y))$, we define $\Re \in \Im(P(X))$ as $\Re(A)=$ $f^{-1}(\bar{\Re})(A)=\bar{\Re}(f(A))$.

Then $[K(\Re, X)]=[K(\bar{\Re}, f(X))]$ and $\left[\bar{\Re} \subseteq \sigma_{R}\right] \otimes$ $\left[C_{R}(f)\right] \leq\left[\Re \subseteq \tau_{R}\right]$. For any $\wp \leq \Re$, we set $\bar{\wp} \in \Im(P(Y))$ defined as $\bar{\wp}(f(A))=f(\wp)(f(A))=$ 
$\wp(A), \quad A \subseteq X$, and we have $[F F(\wp)] \leq$ $[F F(\bar{\wp})],[K(\bar{\wp}, f(X))] \geq[K(\wp, X)]$. Therefore

$$
\begin{aligned}
& {\left[\Gamma_{R}(X, \tau)\right] \otimes\left[C_{R}(f)\right] \otimes\left[K_{R}^{\prime}(\bar{\Re}, f(X))\right]} \\
& =\left[\Gamma_{R}(X, \tau)\right] \otimes\left[C_{R}(f)\right] \otimes[K(\bar{\Re}, f(X))] \\
& \quad \otimes\left[\bar{\Re} \subseteq \sigma_{R}\right] \\
& \leq\left[\Gamma_{R}(X, \tau)\right] \otimes\left[\Re \subseteq \tau_{R}\right] \otimes[K(\Re, X)] \\
& =\left[\Gamma_{R}(X, \tau)\right] \otimes\left[K_{R}(\Re, X)\right] \\
& \leq[(\exists \wp)((\wp \leq \Re) \wedge K(\wp, X) \otimes F F(\wp))] \\
& \leq[(\exists \wp)((\wp \leq \Re) \wedge K(\bar{\wp}, f(X)) \otimes F F(\bar{\wp}))] \\
& \leq\left[\left(\exists \wp^{\prime}\right)\left(\left(\wp^{\prime} \leq \bar{\Re}\right) \wedge K\left(\wp^{\prime}, f(X)\right) \otimes F F\left(\wp^{\prime}\right)\right)\right],
\end{aligned}
$$

where $K_{R}^{\prime}$ is related to $\sigma$. Therefore

$$
\begin{aligned}
& {\left[\Gamma_{R}(X, \tau)\right] \otimes\left[C_{R}(f)\right]} \\
& \leq K_{R}^{\prime}(\bar{\Re}, f(X)) \longrightarrow\left(\exists \wp^{\prime}\right)\left(\left(\wp^{\prime} \leq \bar{\Re}\right)\right. \\
& \left.\quad \wedge K\left(\wp^{\prime}, f(X)\right) \otimes F F\left(\wp^{\prime}\right)\right) \\
& \leq \bigwedge_{\bar{\Re} \in \Im(P(Y))}\left(K _ { R } ^ { \prime } ( \overline { \Re } , f ( X ) ) \longrightarrow ( \exists \wp ^ { \prime } ) \left(\left(\wp^{\prime} \leq \bar{\Re}\right)\right.\right. \\
& \left.\left.\quad \wedge K\left(\wp^{\prime}, f(X)\right) \otimes F F\left(\wp^{\prime}\right)\right)\right) \\
& =\left[\Gamma_{R}(f(X))\right] . \square
\end{aligned}
$$

The following theorem gives the image of fuzzifying compact space under fuzzifying almost continuous function.

Theorem 3.3. Let $(X, \tau)$ and $(Y, \sigma)$ be any two fuzzifying topological space and $f \in Y^{X}$ be surjection. Then $=\Gamma(X, \tau) \otimes A C(f) \longrightarrow \Gamma_{R}(f(X))$.

Proof. From the proof of Theorem 3.1 we have for any $\bar{\Re} \in \Im(P(Y))$, we define $\Re \in \Im(P(X))$ as $\Re(A)=$ $f^{-1}(\bar{\Re})(A)=\bar{\Re}(f(A))$.

Then $[K(\Re, X)]=[K(\bar{\Re}, f(X))]$ and $\left[\bar{\Re} \subseteq \sigma_{R}\right] \otimes$ $[A C(f)] \leq[\Re \subseteq \tau]$. For any $\wp \leq \Re$, we set $\bar{\wp} \in \Im(P(Y))$ defined as $\bar{\wp}(f(A))=f(\wp)(f(A))=\wp(A), A \subseteq X$. and we have $[F F(\wp)] \leq[F F(\bar{\wp})], \quad[K(\bar{\wp}, f(X))] \geq$ $[K(\wp, X)]$. Therefore where $K_{R}^{\prime}$ is related to $\sigma$. Therefore

$$
\begin{aligned}
& {[\Gamma(X, \tau)] \otimes[A C(f)] } \\
\leq & K_{R}^{\prime}(\bar{\Re}, f(X)) \longrightarrow\left(\exists \wp^{\prime}\right)\left(\left(\wp^{\prime} \leq \bar{\Re}\right)\right. \\
& \left.\wedge K\left(\wp^{\prime}, f(X)\right) \otimes F F\left(\wp^{\prime}\right)\right) \\
\leq & \bigwedge_{\bar{\Re} \in \Im(P(Y))}\left(K _ { R } ^ { \prime } ( \overline { \Re } , f ( X ) ) \longrightarrow ( \exists \wp ^ { \prime } ) \left(\left(\wp^{\prime} \leq \bar{\Re}\right)\right.\right. \\
& \left.\left.\wedge K\left(\wp^{\prime}, f(X)\right) \otimes F F\left(\wp^{\prime}\right)\right)\right) \\
= & {\left[\Gamma_{R}(f(X))\right] . \square }
\end{aligned}
$$

\section{Conclusion}

The present paper investigates topological notions when these are planted into the framework of Ying's fuzzifying topological spaces (in Lukasiewicz fuzzy logic). It continue various investigations into fuzzy topology in a legitimate way and extend some fundamental results in general topology to fuzzifying topology. An important virtue of our approach (in which we follow Ying) is that we define topological notions as fuzzy predicates (by formulae of Łukasiewicz fuzzy logic) and prove the validity of fuzzy implications (or equivalences). Unlike the (more widespread) style of defining notions in fuzzy mathematics as crisp predicates of fuzzy sets, fuzzy predicates of fuzzy sets provide a more genuine fuzzification; furthermore the theorems in the form of valid fuzzy implications are more general than the corresponding theorems on crisp predicates of fuzzy sets. The main contributions of the paper are to study nearly compact space in fuzzifying topology and the behavior of (nearly) compact spaces under various types of mappings. There are some problems for further study:

(1) What is the justification for fuzzifying near compactness in the setting of $(2, L)$ topologies.

(2) Obviously, fuzzifying topological spaces in [16] form a fuzzy category. Perhaps, this will become a motivation for further study of the fuzzy category.

(3) It would be interesting to find results on productivity.

\section{References}

[1] C.L. Chang, "Fuzzy topological spaces", J. Math. Anal. Appl., 24, pp. 182-190, 1968.

$[\Gamma(X, \tau)] \otimes[A C(f)] \otimes\left[K_{R}^{\prime}(\bar{\Re}, f(X))\right]$

[2] J.A. Goguen, "The fuzzy Tychonoff Theorem", J. Math. Anal. Appl., 43, pp. 182-190, 1973.

[3] U.Höhle, "Upper semicontinuous fuzzy sets and applications”, J. Math. Anal. Appl., 78, pp.659-673, 1980.

[4] U.Höhle, Many Valued Topology and its Applications, Kluwer Academic Publishers, Dordrecht, 2001. 
[5] U. Höhle and S. E., Rodabaugh, Mathematics of Fuzzy Sets : Logic, Topology, and Measure Theory, in : Hand-book of Fuzzy Sets Series, vol. 3, Kluwer Academic Publishers, Dordrecht, 1999.

[6] U. Höhle and A.Šostak, Axiomatic foundations of fixed-basis fuzzy topology. In : U.H“ohle and S.E. Rodabaugh, 1999. (Eds.), Mathematics of Fuzzy Sets : Logic, Topology, and Measure Theory, in : Handbook of Fuzzy Sets Series, vol. 3, Kluwer Academic Publishers, Dordrecht, pp.123-272, 1999

[7] U. Höhle and S.E., Rodabaugh and A.Sostak, (Eds.), "Special Issue on Fuzzy Topology", Fuzzy Sets and Systems, 73, 1--'183, 1995.

[8] J. L. Kelley, General Topology, Van Nostrand, NewYork 1995.

[9] T. Kubiak, On Fuzzy Topologies, Ph.D. Thesis, Adam Mickiewicz University, Poznan, Poland 1985.

[10] Y. M. Liu and M. K. Luo, Fuzzy Topology, World Scientific, Singapore, 1998.

[11] S. E. Rodabaugh, Categorical foundation of variablebasis fuzzy topology, in : U. Höhle and S. E. Rodabaugh, (Eds.), Mathematics of Fuzzy Sets : Logic, Topology, and Measure Theory, in : Handbook of Fuzzy Sets series, vol. 3, Kluwer Academic Publishers, Dordrecht, pp. 273-388, 1999.

[12] J. B. Rosser and A. R. Turquette. Many-Valued Logics, North-Holland, Amsterdam 1952.

[13]M. K. Singal and A. Mathur, "On nearly compact spaces”, Boll. Un. Math. Ita,. vol. 4, no. 2, pp.702-710, 1969.

[14]M. K. Singal and A. "Mathur, Onnearly compacts pacesII”, Boll.Un. Math. Ital. 9, pp. 670-678, 1974.

[15]G. J. Wang, Theory of L-Fuzzy Topological Spaces, Shanxi Normal University Press, Xi'an, inChinese, 1988.

[16]M. S. Ying, “A new approach for fuzzy topology (I), (II), (III)", Fuzzy Sets and Systems, 39, 47, 55, pp. 303321, pp. 221-232, pp. 139-207, 1991-1993.
[17] M. S. Ying, "Compactness in fuzziying topology", Fuzzy Sets and Systems, 55, pp. 79-92, 1993.

[18]A. M. Zahran, "Almost continuity and $\delta$-continuity in fuzzifying topology”, Fuzzy Sets and Systems, 116, pp.339-0352, 2000.

[19] A. M. Zahran, O. R. Sayed, A. K. Mousa, “Completely continuous functions and R-map in fuzzifying topological space", Fuzzy Sets and Systems, 158, pp. 409-423, 2007.

\section{A.M. Zahran}

M.Sc: 1986

Ph.D: 1990

Assoc. Professor: 1996

Professor: 2002

Department of Mathematics, Faculty of Science, Al-Azhar University, Assiut 71524, Egypt

Research Area: Fuzzy topology, General topology

E-mail : amzahran@yahoo.com

\section{O. R. Sayed}

M.Sc: 1995

Ph.D: 2002

Assoc. Professor: 2008

Department of Mathematics, Faculty of Science, Assiut University, Assiut 71516, Egypt

Research Area: Fuzzy topology, General topology

E-mail : o_r_sayed@yahoo.com

\section{Azab Abd-Allah}

M.Sc: 1986

Ph.D: 1991

Assoc. Professor: 2005

Department of Mathematics, Faculty of Science, Assiut University, Assiut 71516, Egypt

Research Area: Fuzzy topology, General topology

E-mail : mazab57@yahoo.com

\section{A.K. Mousa}

M.Sc: 2006

Department of Mathematics, Faculty of Science, Al-Azhar University, Assiut 71524, Egypt

Research Area: Fuzzy topology, General topology

E-mail: a_k_musa@yahoo.com 